\section{Military Technical College Kobry El-Kobbah, Cairo, Egypt}

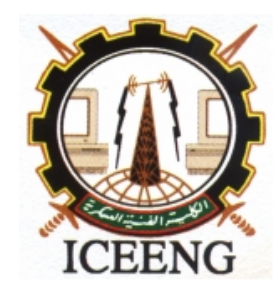

\author{
$8^{\text {th }}$ International Conference \\ on Electrical Engineering \\ ICEENG 2012
}

\title{
The effect of Radio Resource Management in the performance of Heterogeneous Cellular Networks
}

\author{
By
}

Dr. Tarek M. Attia*

\section{Abstract:}

Comparing market estimates for wireless personal communication and considering recent wide band multimedia services with the existing spectrum allocations for these types of systems show that spectrum resource management remains a very important topic.

The coexistence of different cellular networks in the same geographical area requires joint radio resource management (JRRM) for efficient radio resource utilization and enhancement of quality of service (QoS) provisioning, maintain the planned coverage area and provide high capacity.

This paper intends to provide an overview of the RRM problem and envisaged solutions in a Beyond $3 \mathrm{G}$ framework. Different scenarios of call admission control in heterogeneous cellular networks are analyzed and compared. Further, we focus on joint call admission control (JCAC) algorithms in heterogeneous cellular networks and present a JCAC model and assumptions for evaluating and discussing the performance of the JCAC algorithm through simulation results.

\section{Keywords:}

Radio resource management, cellular networks

* National Telecom Regulatory Authority (NTRA), Egypt 


\section{Introduction:}

Recent systems require much higher data rates than current systems. Data rates in wireless personal communication systems are certainly limited by propagation conditions such as multipath, but the primary constraining factor is the link budget (i.e., terminal power consumption). Since the required transmitter power increases linearly with the bandwidth, high-speed radio accesses have only a very limited range [1].

As mobile networks evolve to offer both circuit and packet-switched services, users will be connected permanently via their personal terminal of choice to the network. With the development of intelligence in core network $(\mathrm{CN})$, both voice and broadband multimedia traffic will be directed to their intended destination with reduced latency and delays. As the number of IP-based mobile applications grows, 3G systems offer the most flexible access technology because it allows for mobile, office, and residential use in a wide range of networks.

Wireless systems beyond 3G (e.g., 4G) will consist of a layered combination of different access technologies including Cellular systems (e.g., existing 2G and 3G systems) for wide area mobility, Worldwide interoperability for microwave access (WiMAX) IEEE 802.16 for metropolitan areas, wireless local area networks (WLANs) for dedicated indoor applications (such as IEEE 802.11x) and wireless personal area networks (WPANs) for short-range and low mobility applications around a room in the office or at home [2]. These access systems will be connected via a common IP-based core network that will also handle interworking between the different systems.

The 4G networks will be all-IP-based heterogeneous networks that will allow users to use any system at anytime and anywhere. Users carrying an integrated terminal can use a wide range of applications provided by multiple wireless networks. At the same time, a low per-bit transmission cost will be maintained by improved spectral efficiency of the system. With reconfigurable terminals becoming available, a mobile network operator is in the position to offer a multi-system access to ensure that users are "always best connected". An "integrated operator" can offer a seamless personalized access to mobile multimedia applications charged together over one bill of the same operator and will be motivated to use JRRM techniques for the optimized usage of the radio resources of the different radio access technologies (RATs) [3].

JRRM concepts and algorithms have to meet requirements from the viewpoint of overall network performance, the individual user's point of view and the operator's point of view. JRRM solutions should integrate all these aspects.

A motivation for heterogeneous cellular networks arises from the fact that no single RAT can provide ubiquitous coverage and continuous high QoS levels across multiple smart spaces (e.g. home, office, public smart spaces, etc). Moreover, increasing users' demand for advanced services that consume a lot of network resources has made network researchers developed more and more spectrally efficient multiple access and modulation schemes to support these services. Consequently, wireless networks have 
evolved from one generation to another. However, due to huge investment in existing RATs, operators do not discard their existing RATs when they acquire new ones [4]. In heterogeneous cellular networks, radio resources are managed by using algorithms such as joint call admission control algorithms, joint scheduling algorithms, joint power control algorithms, load balancing algorithms, etc.

This paper is organized as follows. In section 2, an overview of RRM is given. In section 3, joint call admission control (JCAC) in heterogeneous cellular network is described. In section 4, we present a JCAC model and assumptions. In section 5, simulations results are given to show the effectiveness of call admission control in the different scenarios.

\section{Overview of Radio Resource Management (RRM):}

The coexistence of different cellular networks in the same geographical area entails JRRM for enhanced QoS provisioning and efficient radio resource utilization. The concept of JRRM arises in order to efficiently manage the common pool of radio resources that are available in each of the existing RATs [5]. In heterogeneous cellular networks, the radio resource pool consists of resources that are available in a set of cells, typically under the control of a radio network controller or a base station controller.

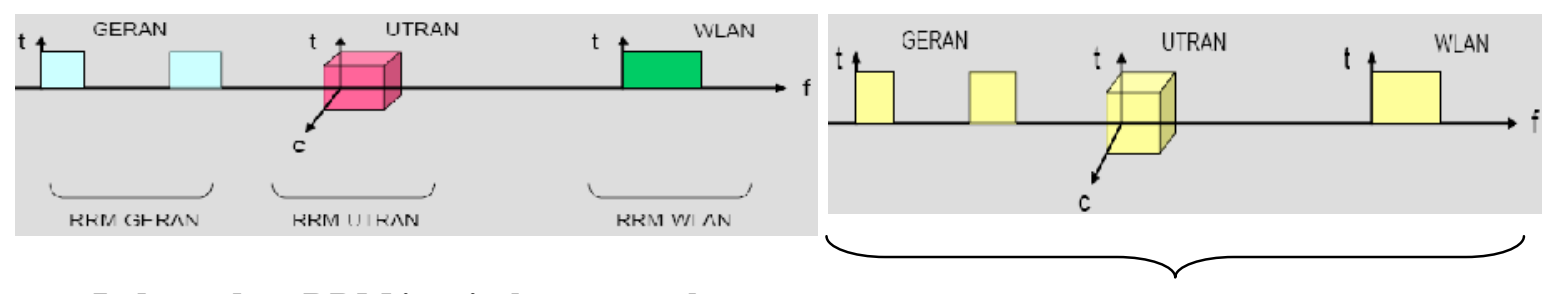

Independent RRM in wireless networks

Joint RRM in wireless networks

Figure (1): Independent RRM and Joint RRM in heterogeneous wireless networks

In heterogeneous cellular networks, radio resource can be independently managed or jointly managed as shown in figure(1). With joint radio resource management in heterogeneous cellular networks, mobile users will be able to communicate through any of the available RATs and roam from one RAT to another, using multi-mode terminals(MTs) [ 6,7,8,9].

An efficient dynamic RRM scheme that consider the cooperation of two or more protocol layers have been used to optimise the system usage in run time to guarantee good performance of the overall system by maximizing some performance indicators, such as the overall network throughput, the resource utilization and total network earning, and at the same time to minimize other indicators, such as end-to-end delay, packet discard rate, call dropping rate and signal-to-noise ratio. RRM schemes can include a set of service control functions, which are categorised into network based functions and connection based functions. Network based functions include admission 
control, load control, packet scheduler and resource manager; whereas connection based functions include power control and handover control[10].

\section{Joint Call Admission Control (JCAC) in heterogeneous cellular networks:}

JCAC algorithm is one of the JRRM algorithms, which decides whether an incoming call can be accepted or not. It also decides which of the available radio access networks is most suitable to accommodate the incoming call. Call admission control (CAC) procedure in heterogeneous cellular networks are shown in figure(2).

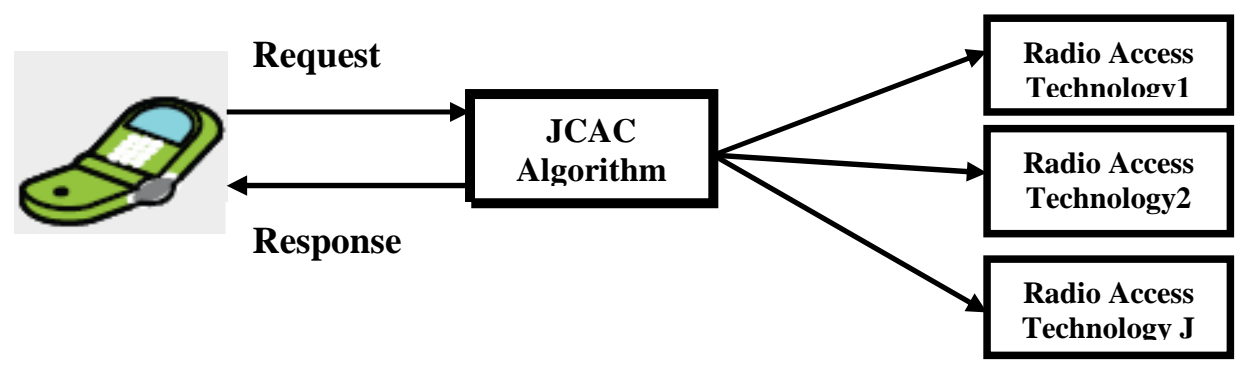

Figure (2): Procedure of call admission control in heterogeneous cellular networks

When a multi-mode mobile terminal wanting to make a call, it will send a request to the JCAC algorithm. The JCAC scheme will execute the JCAC algorithm and then select the most suitable RAT for the incoming call.

A number of RAT selection approaches have been proposed for JCAC algorithms in heterogeneous cellular networks. These approaches can be classified as single criteria or multiple-criteria. Multiple-criteria of JCAC algorithms make RAT selection decisions based on a combination of multiple criteria( such as network load, service cost, service class, random selection, path loss measurement and RAT layer).

\subsection{Bandwidth allocation techniques:}

In order to give different levels of priorities to different calls in wireless networks, it may be necessary to allocate certain block of basic bandwidth units (bbu) for new and handoff calls as well as for different classes of calls such as voice, video, etc,. Bandwidth allocation strategies for wireless networks can be classified into four sets including complete sharing, complete partitioning, handoff call prioritization and service class prioritization.

\subsubsection{Complete sharing:}

Complete sharing scheme is the simplest bandwidth allocation policy. It is a nonprioritization scheme in which new and handoff calls are treated by the same way. An incoming call is accepted as long as there is an enough radio resource to accommodate it. When the network gets to its maximum capacity, a new call will be blocked while a handoff call will be dropped. Two major advantages of complete sharing CAC scheme are good radio resource utilization and simplicity of implementation. However, it has a high handoff call dropping probability because it does not give preference to any call 
and has a poor of QoS performance[11]. Figure(3) is the state transition diagram for complete sharing scheme where $\lambda n$ represents new call arrival rate, $\lambda h$ represents handoff call arrival rate, $n$ represents new call departure rate and $h$ represents handoff call departure rate.

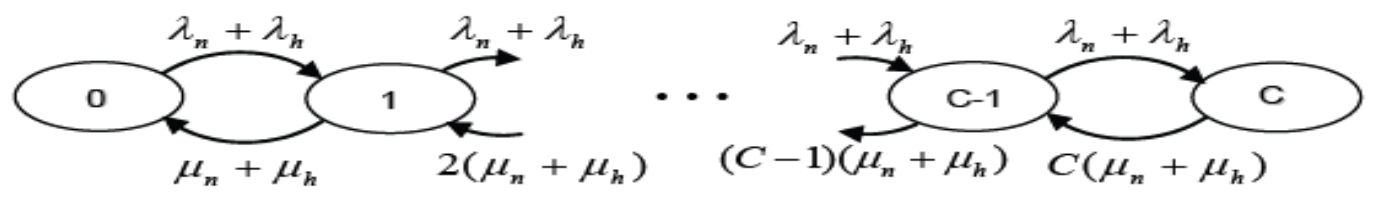

Figure (3): State transition diagram for complete sharing policy

\subsubsection{Complete partitioning:}

All the available bandwidth is partitioned into pools. Each pool is assigned to a particular traffic class of calls and/or particular type of calls (new or handoff calls). An incoming call is admitted if there is an available channel in the pool allocated for the class of the incoming call. This policy allocates a fixed bandwidth $\mathrm{C} 1$ to service $\mathrm{S} 1$ and a fixed bandwidth $\mathrm{C} 2$ to service $\mathrm{S} 2$ such that $(\mathrm{C} 1+\mathrm{C} 2)$ is less than or equal $\mathrm{C}$. This is a case of two independent queues, and the blocking probability is given by the well known Erlang-B formula.

\subsection{Handoff call prioritization:}

Due to mobility of users within the coverage area of wireless networks, an accepted call that has not been completed in the current cell has to be handed over to another cell. The call may not be able to get a channel in the new cell to continue its service due to limited radio resources in wireless networks and it may be dropped.

In order to ensure that handoff call dropping probability is kept below a certain level, handoff calls are usually admitted with a higher priority compared with new calls. Handoff call prioritization has an advantage of low handoff call dropping probability. However, the advantage of low handoff call probability is at the expense of new call blocking probability, which is high. Several handoff-priority schemes have been proposed in the literature and some of these are summarized as follows:

\subsubsection{Guard Channel:}

Some channels are specifically reserved in each cell to take care of handoff calls. For example, if the total number of available channels in a single cell is $\mathrm{C}$ and the number of guard channels is $\mathrm{G}$, a new call is accepted if the total number of channels used by ongoing calls are less than the threshold $(\mathrm{H}=\mathrm{C}-\mathrm{G})$, whereas a handoff call is always accepted if there is an available channel[12]. Guard channel scheme can be divided into two categories namely static and dynamic strategies. In static guard channel scheme, the value of $\mathrm{H}$ is constant whereas in dynamic guard channel scheme, $\mathrm{H}$ varied with the arrival rates of new and handoff calls. Figure(4) shows the state transition diagram for a single-class service using guard channel scheme. 


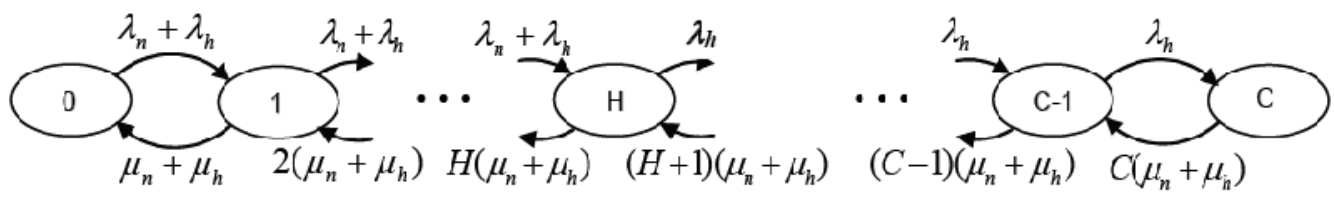

Figure (4): State transition diagram for guard channel scheme

\subsubsection{Fractional Guard Channel:}

Handoff calls are prioritized over new calls by accepting an incoming new call with a certain probability that depends on the number of busy channels. In other words, when the number of busy channels becomes larger, the acceptance probability for a new call becomes smaller, and vice versa. This approach helps to reduce the handoff call dropping probability. The policy has a threshold, $\mathrm{H}$ for limiting the acceptance of new calls. A handoff is accepted as long as there is a channel available. Before the wireless system gets to threshold, new calls are accepted with a probability of 1 . After threshold, a new call is accepted with a probability of $\alpha p$ where $0 \leq \alpha p \leq$ and $\mathrm{G}<\mathrm{p}<\mathrm{C}$. New calls are rejected when the system reaches the maximum capacity.

\subsubsection{Queuing Priority Scheme:}

Queuing priority scheme accepts calls (new and handoff) whenever there are free channels. When all the channels are occupied, handoff calls are queued while new calls are blocked or all incoming calls are queued with certain rearrangement in the queue.

When radio resource becomes available, one or some of the calls in the handoff queue are served until there is no more resource. The remaining calls are queued until resource becomes available again. However, a call is only queued for a certain period of time. If radio resource is not available within this period, the call will be dropped.

The main disadvantage of queuing priority scheme needs a lot of buffers to deal with real-time multimedia traffic and also needs a sophisticated scheduling mechanism in order to meet the QoS requirements of delay-sensitive calls[13].

\subsubsection{QoS Degradation Scheme:}

QoS degradation can either be delay degradation or bandwidth degradation. In delay degradation method, the amount of radio resources allocated to non-real-time services is reduced during network congestion. When a handoff call arrives and there is no radio resource to accommodate the handoff call. Some non-real-time services are degraded to free some bandwidth, which is used to accommodate the incoming handoff call.

In bandwidth degradation method, calls are categorized as degradable and nondegradable calls. Degradable calls have flexible QoS requirements. For most multimedia applications service can be degraded temporarily as long as it is still within the pre-defined range. Bandwidth degradation reduces handoff call dropping by reducing the bandwidth of the ongoing adaptive calls during network congestion. When a handoff call arrives and there is network congestion, the system is able to free some radio resource to admit the handoff calls by degrading some of the ongoing adaptive 
calls.

\subsection{Service-class prioritization:}

In wireless systems which support multiple service classes, the limited bandwidth has to be shared among the multiple traffic classes. However, one major challenge in the design of CAC policy is to provide privilege treatment among users of different service classes while still utilizing the system resources efficiently. Privilege treatments are given to certain classes of calls due to some subscribers in a particular service class are willing to pay more for better QoS or some calls (such as voice call) have rigorous QoS requirements and therefore require privilege treatment.

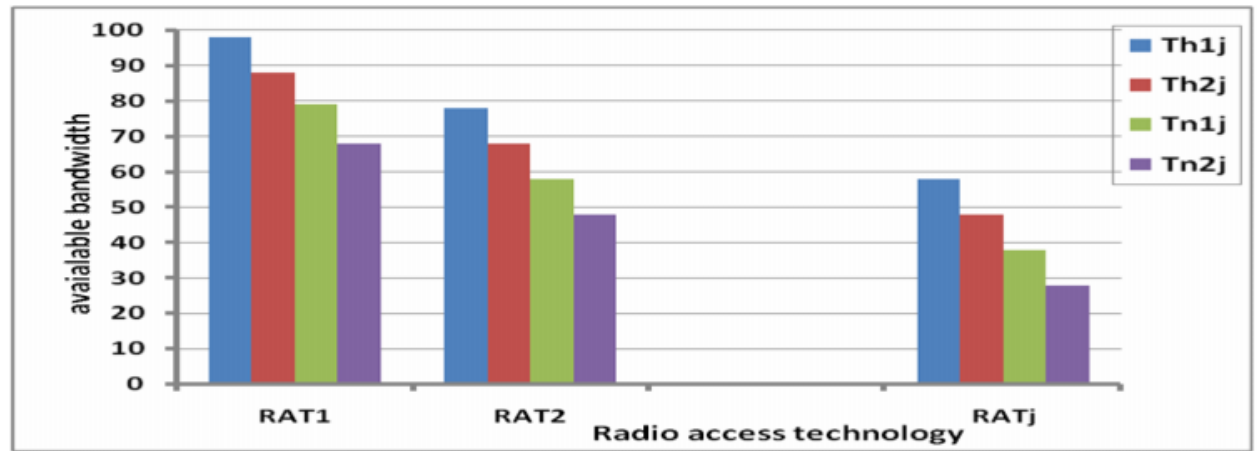

Figure(5):Prioritization scheme in a two-class of heterogeneous wireless network Figure(5) shows the prioritization scheme used in this paper. As shown in the figure, the two class J-RAT heterogeneous wireless network (where $\mathrm{J}$ is the total number of RATs in the network) has different thresholds for prioritizing the two classes of calls. Tn1j and $\mathrm{Tn} 2 \mathrm{j}$ are the thresholds for rejecting class- 1 and class- 2 new calls in $\mathrm{RAT} \mathrm{j}$, respectively whereas Th1 $\mathrm{j}$ and $\mathrm{Th} 2 \mathrm{j}$ are the thresholds for rejecting class- 1 and class -2 handoff calls in RATj, respectively. It can be seen that handoff calls are prioritized over new call by using higher thresholds for handoff call.

It can also be seen that class- 1 calls (voice service) are prioritized over class- 2 calls (Video services). The rejection thresholds can be static or dynamic. Static thresholds are very simple to implement but are less efficient whereas dynamic threshold are more efficient but are more complicated.

Bandwidth allocation to individual calls in cellular networks can be static or adaptive. In static bandwidth allocation, a fixed unit of radio resource is allocated to each call, and the allocated unit is fixed during the entire duration of the call. In adaptive bandwidth allocation, resource allocated to each call varies between a minimum value and a maximum value. When the network is underutilized, maximum amount of radio resources are allocated to certain type of calls (adaptive calls). However, when the network is being oversubscribed, minimum amount of radio resources are allocated to adaptive calls in order to free up some amount of radio resources to accommodate more calls. Adaptive bandwidth allocation improves radio resource allocation efficiency but it is more complicated. They also incur more signaling overhead. 


\section{Modeling of JCAC algorithm in heterogeneous cellular networks:}

We present a model of a load-based JCAC algorithm in a two-RAT heterogeneous cellular network supporting two classes of calls: the voice call (class-1) and the video call (class-2). The load based-JCAC algorithm admits an incoming call into the least loaded RAT in the heterogeneous wireless networks (scenarios 1 and 2 in Table 1). We also consider independent call admission control (ICAC) where radio resources are independently managed in the two RATs (scenarios 3 and 4 in Table 1). The four scenarios considered in the simulations are summarized in Table1.

Scenarios 2 and 4 uses fixed bandwidth allocation where fixed amount of bandwidth (full rate) is allocated to class-1 calls and fixed amount of bandwidth (maximum rate) is allocated to class- 2 calls at all times.

Table(1): Four scenarios considered in the simulations

\begin{tabular}{|l|l|l|}
\hline Scenarios & Bandwidth Allocation for Class1 and Class2 & Resource Management \\
\hline Scenario1 (AJCAC) & Adaptive Allocation Call Admission control & Joint \\
\hline Scenario2 (FJCAC) & Fixed Allocation Call Admission control & Joint \\
\hline Scenario3 (AICAC) & Adaptive Allocation Call Admission control & Independent \\
\hline Scenario4 (FICAC) & Fixed Allocation Call Admission control & Independent \\
\hline
\end{tabular}

Scenarios 1 and 3 use adaptive bandwidth allocation where full rate bandwidth is allocated to class- 1 calls when the network is underutilized whereas half rate bandwidth is allocated to class- 1 calls when the networks is over subscribed. Similarly, class-2 calls are allocated a maximum amount of bandwidth when the network is underutilized whereas a minimum amount of bandwidth is allocated when the network is oversubscribed.

\subsection{System model and assumptions:}

We consider a generic heterogeneous cellular network, which consists of $\mathbf{J}$ number of RATs with co-located cells. Cellular networks such as GSM, GPRS, UMTS, LTE, etc, can have the same and fully overlapped coverage, which is technically feasible, and may also save installation cost[14].

We consider cases where radio resources are independently or jointly managed in the heterogeneous network and each cell in $\operatorname{RATj}(j=1, \ldots, J)$ has a total of Bj basic bandwidth units (bbu). The physical meaning of a unit of radio resources (such as time slots, code sequence, etc) is dependent on the specific technological implementation of the radio interface. However, no matter which multiple access technology (TDMA, FDMA, CDMA, or OFDMA) is used, we could interpret system capacity in terms of effective or equivalent bandwidth. Therefore, whenever we refer to the bandwidth of a call, we mean the number of basic bandwidth units that is adequate for guaranteeing the desired QoS for this call, which is similar to the approach used for wireless networks in [7]. 
Our approach is based on decomposing a heterogeneous cellular network into groups of collocated cells and there are overlapping cells form a group of co-located cells. A newly arriving call will be admitted into one of the cells in the group of co-located cells where the call is located. If the call cannot be admitted into any of the cells it will be blocked.

Following the general assumption in cellular networks, new and handoff class-i calls arrive in the group of co-located cells according to Poisson process with rate $\lambda_{i}^{n}$ and $\lambda_{i}^{h}$ respectively. The arrival rates of a split Poisson process are also Poisson and the channel holding time for class-i calls is exponentially distributed with mean $1 / \mu_{i}[15]$.

\subsection{Markov model:}

The load-based JCAC algorithm can be modeled as a multi-dimensional Markov chain. The state space of the group of co-located cells can be represented by a $(2 * \mathrm{~K} * \mathrm{~J})$ dimensional vector given as:

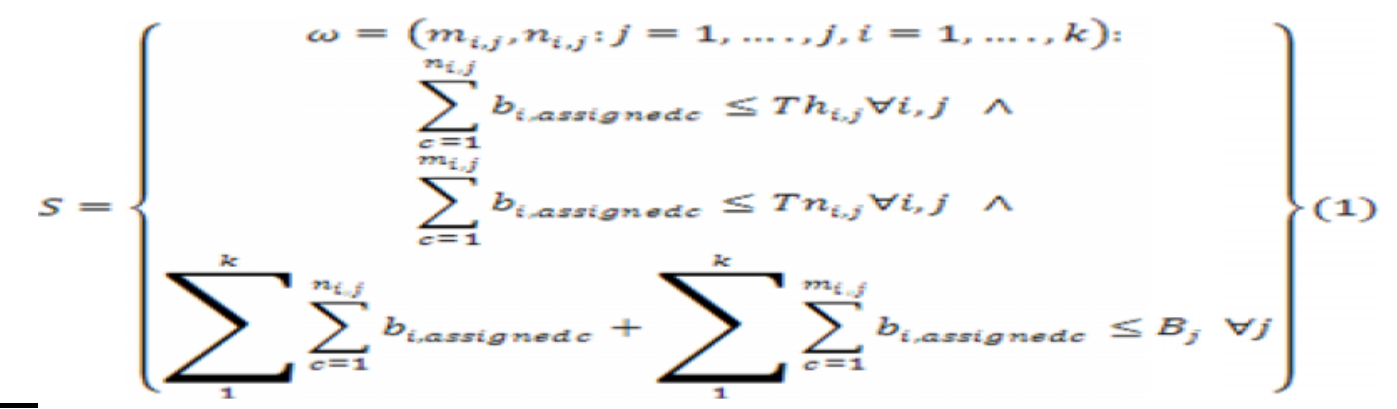

Where $n i, j$ is a non-negative integer denotes the number of ongoing handoff class-i calls in RAT $\mathrm{j}$ and $m i, j$ is a non-negative integer denotes the number of ongoing new class- $\mathrm{i}$ calls in RATj. $S$ denotes the state space of all admissible states of the group of collocated cells. $\mathrm{Bi}$, assigned is the number of basic bandwidth unit (bbu) allocated to an incoming class-i call, and the values vary between bi,min and bi, max.

Let $\rho_{\text {han } i, j}$ and $\rho_{\text {new } i, j}$ denote the load generated by handoff class- $i$ calls and new class $-i$ calls, respectively, in RAT-j. Let $1 / \mu_{i}^{\mathrm{h}}$ and $1 / \mu_{\mathrm{i}}^{\mathrm{n}}$ denote the channel holding time of handoff class-i call and new class-i call respectively, and let $\lambda_{\mathrm{i}, \mathrm{j}}^{\mathrm{h}}$ and $\lambda_{\mathrm{i}, \mathrm{j}}^{\mathrm{n}}$ denote the arrival rates of handoff class-i call and new call of class-i call in RATj, respectively, then

$$
\begin{aligned}
& \rho_{\text {hami, } j}=\frac{\lambda_{, j}^{\mathrm{h}}}{\mu_{\mathrm{h}, j}^{\mathrm{h}}} \forall i, j \\
& \rho_{\text {newi }, j}=\frac{\lambda_{i, j}^{\mathrm{n}}}{\mu_{\mathrm{i}, j}^{\mathrm{n}}} \forall i, j
\end{aligned}
$$

From the steady state solution of the Markov model, performance measures of interest can be determined by summing up appropriate state probabilities. Let $P(s)$ denotes the steady state probability that system is in state $s(s \in S)$. From the detailed balance equation, $P(s)$ is obtained as: 


$$
P(s)=\frac{1}{F} \prod_{i=1}^{\mathrm{k}} \prod_{j=1}^{j} \frac{\left(\rho_{\text {han } i, j}\right)^{n_{i, j}}}{n_{i, j} !} \cdot \frac{\left(\rho_{\text {new } i, j}\right)^{m_{i, j}}}{m_{i, j} !} \forall s \in S
$$

where $\mathrm{F}$ is a normalization constant given by:

$$
F=\sum_{z \in S_{A i}} \prod_{i=1}^{k} \prod_{j=1}^{j} \frac{\left(\rho_{\text {han } i, j}\right)^{m_{i, j}}}{n_{i, j} !} \cdot \frac{\left(\rho_{\text {new } i, j}\right)^{m_{i j}}}{m_{i, j} !}
$$

A handoff class- $i$ call is dropped in the group of collocated cells if none of the RATs in the group of collocated cells has enough bbu to accommodate the handoff call. Let $S_{\mathrm{di}} \subset S$ denote the set of states in which a handoff class- $i$ call is dropped in the group of collocated cells. Thus the handoff call dropping probability for a class- $i$ call, $P_{d \mathrm{i}}$, in the group of co-located cells is given by:

$$
P_{\mathrm{di}}=\sum_{z \in \mathrm{S}_{\mathrm{A} i}} P(s)
$$

A new class-i call is blocked in the group of co-located cells if none of the RATs in the group of co-located cells has enough bbu to accommodate the new call. Let $S_{\mathrm{bi}} \subset S$ denote the set of states in which a new class-i call is blocked in the group of collocated cells. Thus the new call blocking probability, $P_{\mathrm{bi}}$ for a class-i call in the group of collocated cells is given by:

$$
P_{\mathrm{bi}}=\sum_{s \in \mathrm{S}_{\mathrm{hi}}} P(s)
$$

\section{Numerical results:}

In this section, the performance of the JCAC scheme is evaluated through simulations.

Results for both class- 1 calls and class- 2 calls are presented for the four scenarios shown in Table1. The parameters used in the simulations are $B 1=20, B 2=40$, $\operatorname{Tn} 1,1=\operatorname{Tn} 2,1=12, \operatorname{Th} 1,1=\operatorname{Th} 2,1=20, \quad \operatorname{Tn} 1,2=\operatorname{Tn} 2,2=24, \quad \operatorname{Th} 2,1=\operatorname{Th} 2,2=40, \quad 1=2=0.5$. Some other parameters used are shown in Table2.

\section{Table(2): Simulation Parameters}

\begin{tabular}{|l|l|}
\hline Scenarios & Bandwidth Allocation for different scenarios in Table1 \\
\hline Scenario 1 & $\mathrm{b} 1, \mathrm{~min}=1 \mathrm{bbu}, \mathrm{b} 2, \mathrm{~min}=3 \mathrm{bbu}, \mathrm{b} 1, \mathrm{max}=2 \mathrm{bbu}, \mathrm{b} 2, \mathrm{max}=8 \mathrm{bbu}$ \\
\hline Scenario 2 & $\mathrm{b} 1, \mathrm{~min}=\mathrm{b} 1, \mathrm{max}=2 \mathrm{bbu}, \mathrm{b} 2, \mathrm{~min}=\mathrm{b} 2, \mathrm{max}=8 \mathrm{bbu}$ \\
\hline Scenario 3 & $\mathrm{b} 1, \mathrm{~min}=1 \mathrm{bbu}, \mathrm{b} 2, \mathrm{~min}=3 \mathrm{bbu}, \mathrm{b} 1, \mathrm{max}=2 \mathrm{bbu}, \mathrm{b} 2, \mathrm{max}=8 \mathrm{bbu}$ \\
\hline Scenario 4 & $\mathrm{~b} 1, \mathrm{~min}=\mathrm{b} 1, \mathrm{max}=2 \mathrm{bbu}, \mathrm{b} 2, \mathrm{~min}=\mathrm{b} 2, \mathrm{max}=8 \mathrm{bbu}$ \\
\hline
\end{tabular}

\subsection{Comparison of new call blocking probabilities for the four scenarios:}

Figure(6) shows the variation of the blocking probability of new call of class-1(Pb1) with call arrival rates for the four scenarios. $\mathrm{Pb} 1$ increases with increase in arrival rates for each of the four scenarios. However, the FICAC scheme has the highest call blocking probability whereas the AJCAC scheme has the lowest call blocking probability. Thus joint radio resource management and bandwidth adaptation reduces blocking probability of new call in heterogeneous cellular networks. 


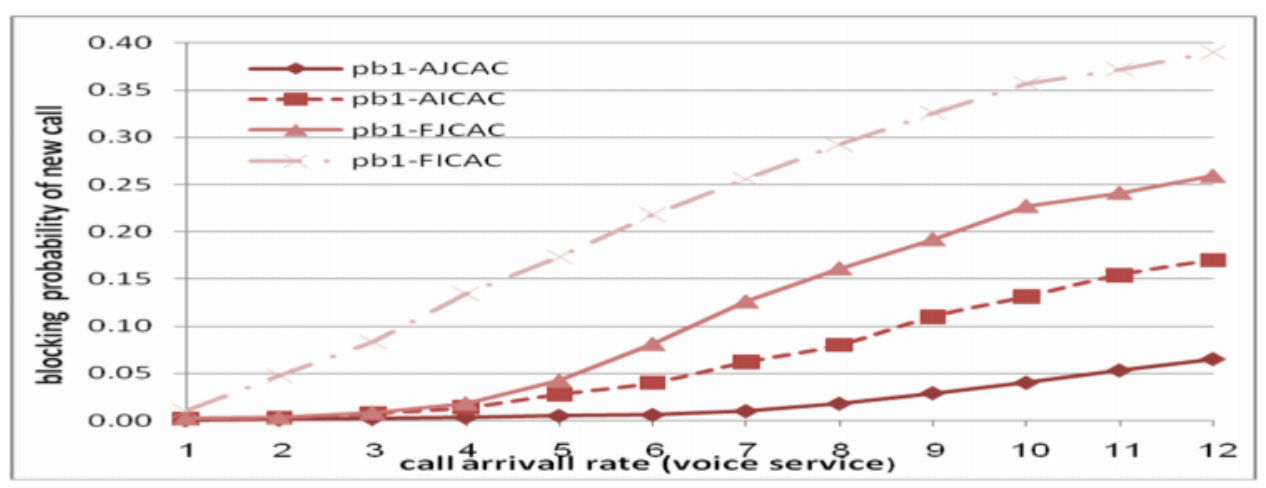

Figure(6): Blocking probability of new call of class-1 against call arrival rate.

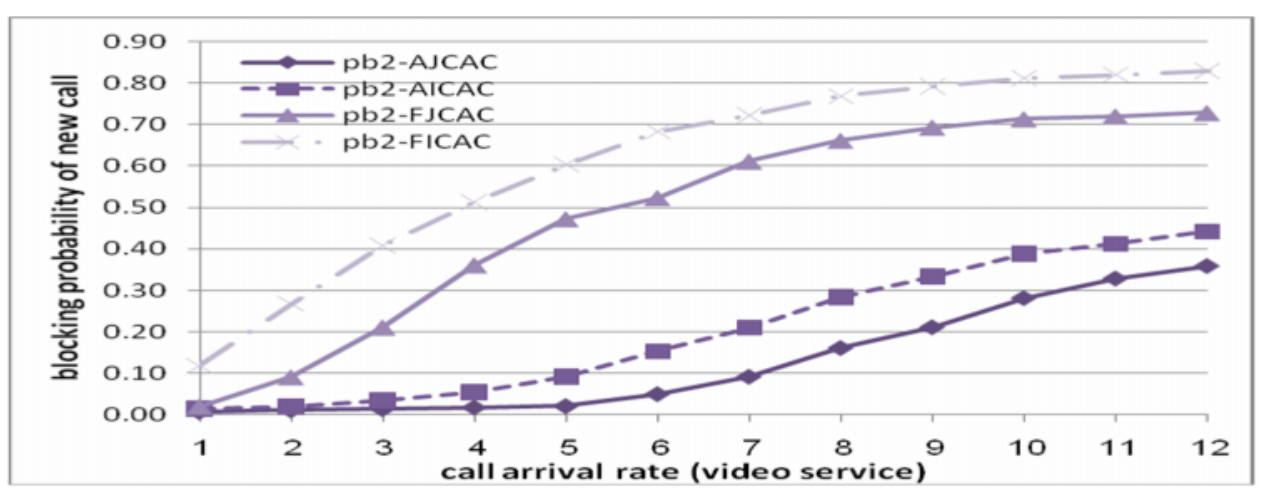

Figure(7): Blocking probability of new call of class-2 against call arrival rate

Figure(7) shows the variation of the blocking probability of new call of class-2 ( $\mathrm{Pb} 2)$ with call arrival rates for the four scenarios. $\mathrm{Pb} 2$ increases with increase in arrival rates for each of the four scenarios. Moreover, $\mathrm{Pb} 2$ in each of the scenarios is higher than the corresponding $\mathrm{Pb} 1$ because class- 2 calls require more bandwidth than class- 1 calls. Thus, it is possible to block a class- 2 call when it is still possible to admit a class- 1 call into the network. However, the FICAC scheme has the highest blocking probability call whereas the AJCAC scheme has the lowest blocking probability of call for class 2 . Both results of figure(6) and figure(7) emphasis that joint radio resource management and bandwidth adaptation reduces blocking probability of new call in heterogeneous cellular networks.

\subsection{Comparison of handoff call dropping probabilities for the four scenarios:}

Figure(8) shows the variation of dropping probability of handoff call class-1 (Pd1) with call arrival rates for the four scenarios. Pd1 increases with increase in arrival rates for each of the four scenarios. However, the AJCAC scheme has the lowest dropping probability call whereas the FICAC scheme has the highest dropping probability call. 


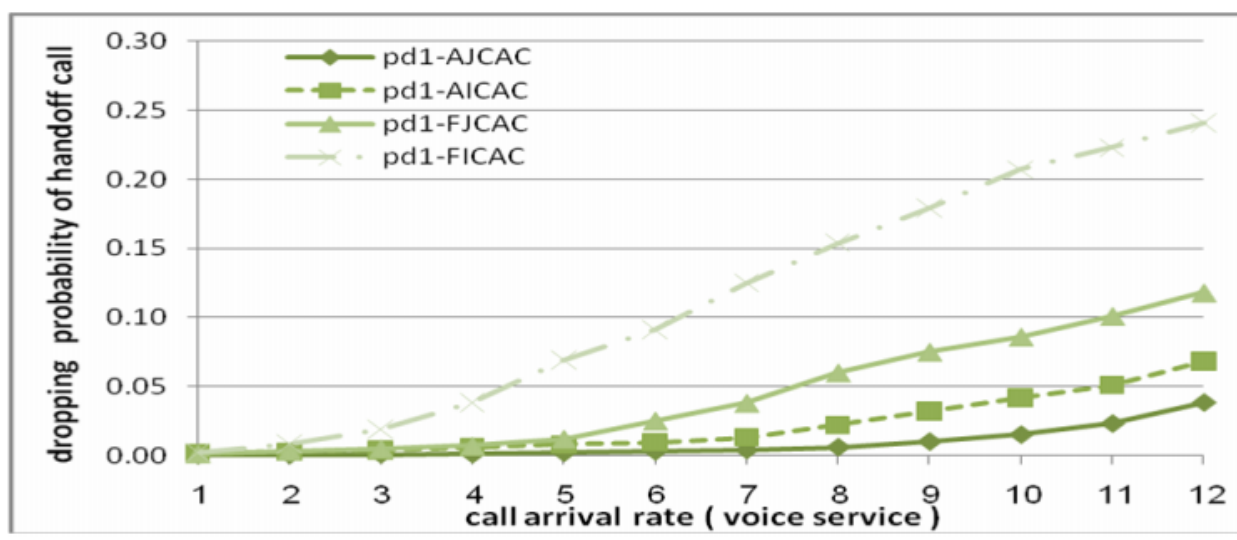

Figure(8): Dropping probability of handoff call of class-1 against call arrival rate

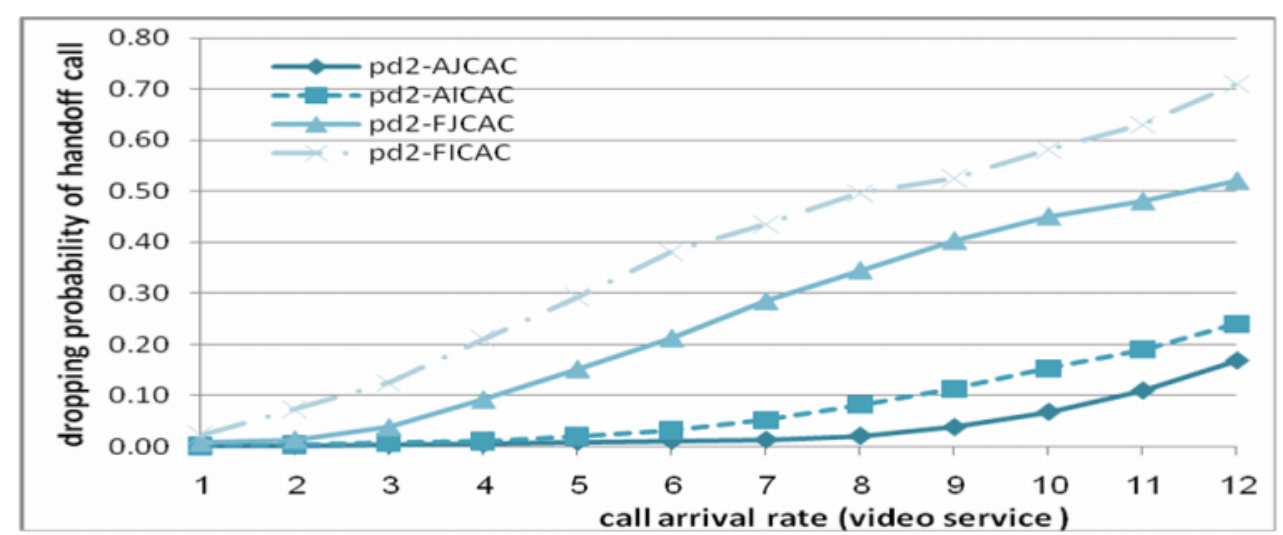

Figure(9): Dropping probability of handoff call of class-2 against call arrival rate

Figure(9) shows a similar trend to figure(8). The AJCAC scheme has the lowest dropping probability for class 2 calls whereas the FICAC scheme has the highest dropping probability call. Both results of figure(8) and figure(9) emphasis that joint radio resource management and bandwidth adaptation reduces dropping probability of handoff call in heterogeneous cellular networks.

\subsection{Comparison of call blocking/dropping probabilities for scenarios 1 and 2:}

Figure(10) compares the blocking probability of new call class-1 and dropping probability of handoff call class-1 for the Fixed and adaptive JCAC schemes. It can be seen that the $\mathrm{Pb} 1$ of AJCAC is greater than the Pd1of AJCAC. Similarly, the Pb1 of FJCAC is greater than the Pd1 of FJCAC. Thus, handoff calls are prioritized over new calls by using the threshold based prioritization scheme shown in figure(5).

Figure(11) compares blocking probability of the new call of class-2(Pb2) and dropping probability of the handoff call of class-2 (Pd2) for the fixed and adaptive JCAC schemes. It can be seen that the $\mathrm{Pb} 2$ of AJCAC is greater than the Pd2 of AJCAC. Similarly, the $\mathrm{Pb} 2$ of FJCAC is greater than of the Pd2 of FJCAC. Thus, handoff calls are prioritized over new calls by using the threshold based prioritization scheme shown in figure(5). 


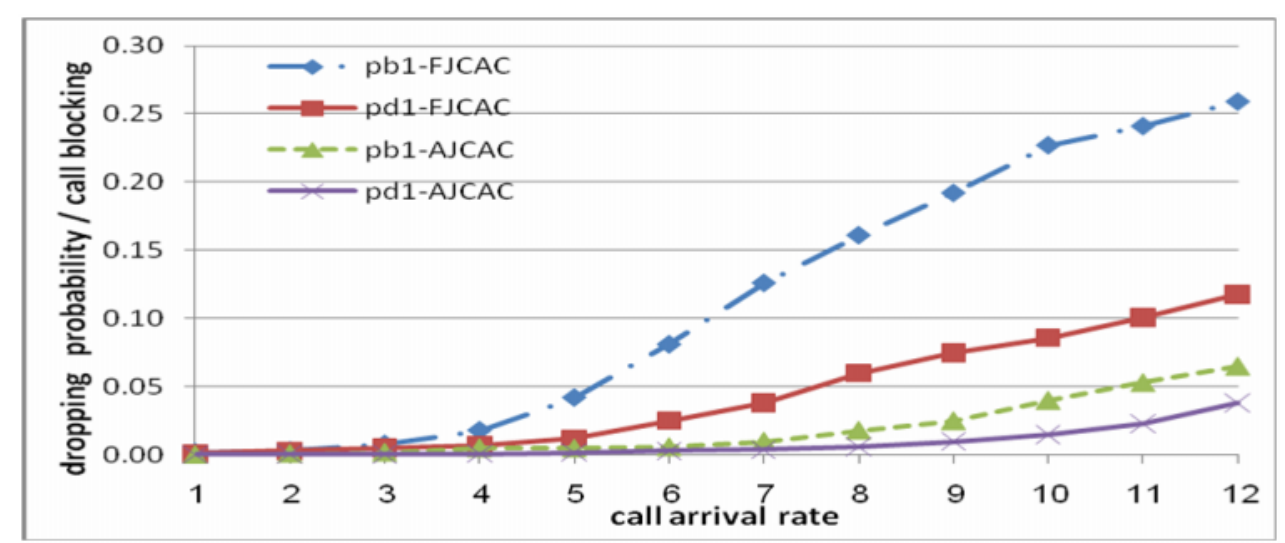

Figure(10):Blocking/Dropping probability call of Class-1 for JCAC schemes

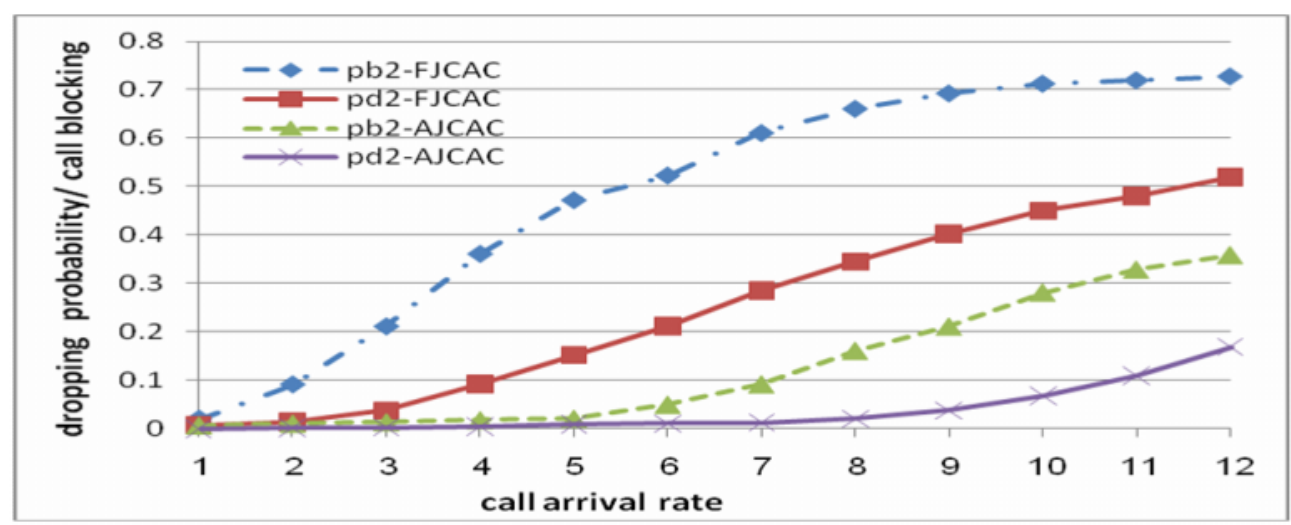

Figure(11): Blocking/Dropping probability of call of Class-2 for JCAC schemes.

\subsection{Comparison of call blocking/dropping probabilities for scenarios 3 and 4:}

Figure(12) compares the blocking probability of new call of class-1 and dropping probability of handoff call of class-1 for the fixed and adaptive ICAC schemes. It can be seen that the $\mathrm{Pb} 1$ of AICAC is greater than the Pd1 of AICAC. Similarly, the Pb1 of FICAC is greater than the Pd1 of FICAC. This is due to handoff calls are prioritized over new calls by using the threshold based prioritization scheme shown in figure(5).

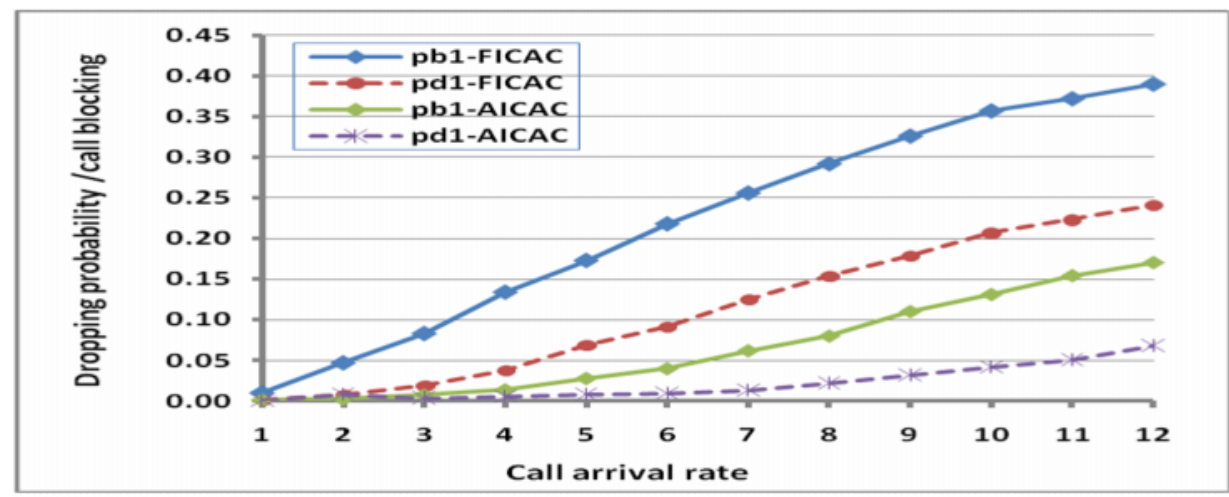

Figure(12): Blocking/Dropping probability of call of Class-1 for ICAC schemes 
Figure(13) compares the blocking probability of new call of class-2 and dropping probability of call of class-2 for the fixed and adaptive ICAC schemes. It can be seen that the $\mathrm{Pb} 2$ of AICAC is greater than the Pd2 of AICAC. Similarly, the Pb2 of FICAC is greater than $\mathrm{Pd} 2$ of FICAC. Thus, handoff calls are prioritized over new calls by using the threshold based prioritization scheme shown in figure(5).

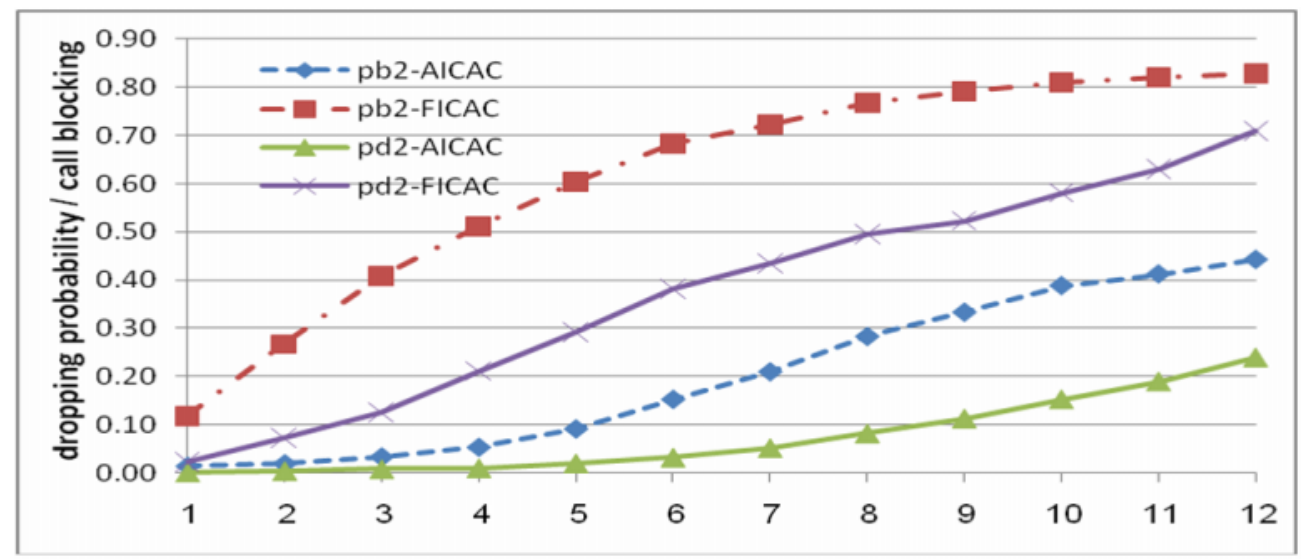

Figure(13): Blocking/Dropping probability of call of Class-2 for ICAC schemes

\section{Conclusion:}

The coexistence of multiple cellular networks in the same geographical area has enabled more efficient utilization of radio resources, enhanced quality of service provisioning, maintain the planned coverage area and offer high capacity through joint radio resource management. A Markov model for a load-based JCAC algorithm has been presented and considering four different scenarios. Simulation results show that joint management of radio resources and bandwidth adaptation reduce call blocking/dropping probability in heterogeneous cellular networks. The usage of call admission control algorithm in heterogeneous cellular networks can guarantee the QoS requirements, minimize call blocking/dropping probability, maximize radio resource utilization, balance distribution of network load, minimize number of vertical handoffs and maximize operators' revenue.

\section{References:}

[1]Jens Zander, Radio Resource Management in Future Wireless Networks" Requirements and Limitations", IEEE Communications Magazine August 1997.

[2]Vijay K. Garg, Wireless Communications and Networking, by Elsevier Inc. Morgan Kaufmann Publishers, 2007.

[3] ITU,WG6;"Cognitive Radio, Spectrum and Radio Resource Management', Wireless World Research Forum, 2004.

[4]Vidales, P.; Baliosian, J.; Serrat, J.; Mapp, G.; Stajano, F.; \& Hopper, A., Autonomic System for Mobility Support in 4G Networks. IEEE Journal on Selected Areas in Communications, Vol. 23, No. 12, December 2005. 
[5] Pérez-Romero, J; Sallent, O.; Agustí, R. \& Díaz-Guerra, M. A, Radio Resource Management strategies in UMTS, John Wiley \& Sons, 2005.

[6] Gelabert, X.; Sallent, O.; \& Agustı', R. , A Markov Approach to Radio Access Technology Selection in Heterogeneous Multiaccess/Multiservice Wireless Networks. IEEE Transactions on Mobile Computing, Vol. 7, No. 10, Oct 2008.

[7] Falowo, O. E.; \& Chan, H. A., Adaptive Bandwidth Management and Joint Call Admission Control to Enhance System Utilization and QoS in Heterogeneous Wireless Networks. EURASIP Journal on Wireless Communications and Networking, Vol. 2007. [8] Lee, S.; Sriram, K.; Kim, Y; \& Golmie, N.; Vertical Handoff Decision Algorithms for Providing Optimized Performance in Heterogeneous Wireless Networks. IEEE Transactions on Vehicular Technology, Vol. 58, No. 2, February 2009.

[9] Niyato, D.; \& Hossain, E., Noncooperative Game-Theoretic Framework for Radio Resource Management in 4G Heterogeneous Wireless Access Networks. IEEE Transactions on Mobile Computing, Vol. 7, No. 3, March 2008.

[10] Farooq Khan, LTE for 4G Mobile Broadband Air Interface Technologies and Performance, Cambridge University Press 2009.

[11] Ho, C. \& Lea, C., Improving Call Admission Policies in Wireless Networks, Wireless Networks, Vol. 5, 1999.

[12] Hong and, D. \& Rappaport, S. S., Traffic Model and Performance Analysis of Cellular Radio Telephony Systems with Prioritized and Non-prioritized Handoff Procedures, IEEE Transaction of Vehicular Technology, Vol. 35, August. 1986.

[13] Chen, H.; Kumar, S. \& Jay Kuo, C.-C. , Dynamic Call Admission Control Scheme for QoS Priority Handoff in Multimedia Cellular Systems, Proceedings of IEEE Wireless Communications and Networking Conference, Orlando, vol. 1, March, 2002, [14] Holma H. \& Toskala, A., WCDMA for UMTS. John Wiley \& Sons, USA, 2001. [15]Orlik, P. V. \& Rappaport, S. S. "On the handover arrival process in cellular communications", ACM/Baltzer Wireless Networks, Vol. 7, No. 2, 2001. 